\title{
New Hemodialysis Access System
}

\author{
Frank Prosl Hans Dietrich Polaschegg ${ }^{\dagger}$ \\ GL Consult, LLC, Duxbury, Mass., USA
}

\section{Key Words}

Hemodialysis • Blood access • Implantable port $\cdot$ Home dialysis · Prophylaxis · Gel lock

\begin{abstract}
A new blood access system for hemodialysis (HD) addresses the major shortcomings of current accessing techniques and helps overcome patient adversity with available choices. The system uses new methods, devices and compositions comprising several novel elements: (1) totally implanted port, elementally simple in design, small and robust with improved safety features, (2) engineered transcutaneous tissue tract (TTT) to precisely guide a tubular conduit to engage the implanted port, (3) viscoelastic gel prophylaxis for internal passages and surface interfaces of the port and TTT, (4) the tool kit aligns the TTT with the port and initiates TTT formation at the time of port placement, (5) an integrated external bloodline circuit comprising the interface between the implanted port and HD machine which can be reused for several treatment sessions. The system provides bloodless, painless, and fail-safe connections with redundant sealing along the complete blood path. It is substantially more robust than current access options and more convenient to use. TTT construct is durable, permanently aligned and fixed to the port enabling non-compromised tissue integrity during access penetration. A bullet-pointed tubular assembly enters and passes through the TTT confining the tubular device to advance towards the port and entering it to engage a solid tactually sensed stop indicating the correct position. The system may be used within a few days of port placement. The access is unobtrusive and preserves patient self-image.
\end{abstract}

Copyright ๑ 2013 S. Karger AG, Basel

\section{KARGER}

Fax +4161306 1234

E-Mail karger@karger.ch

www.karger.com
(C) 2013 S. Karger AG, Basel

0253-5068/13/0353-0072\$38.00/0

Accessible online at: www.karger.com/bpu

\section{Blood Access Development History}

Dr. Scribner's breakthrough innovation in 1960 began life-saving maintenance hemodialysis (HD) enabling millions of people with ESRD added years of life. Dr. Scribner's shunt comprised external silicone rubber/Teflon tubing connected to a vein and artery at the wrist enabling relatively high rates of blood to be withdrawn from the artery and processed by the external dialysis machine and returned to the vein in a continuous cycle. Many other innovations making HD a practical treatment soon followed:

1964: Dr. Shaldon reported using two catheters inserted in the femoral vein providing access for daily home dialysis for acute renal failure which improved patient mortality. Palmer-Quinton reported a peritoneal catheter design. Papers describe the use of an obterated venous catheter for repeated HD.

1965: Arteriovenous fistula (AVF) is described in a paper by Drs. Cimino and Brescia.

1967: Dr. Shaldon introduces home dialysis using an AVF with 5 patients and helpers performing treatment. One patient self-accessed.

1968: Dr. McMillon warns that AVF markedly increases circulatory load on the heart.

1973: Matsumote reports excellent patency in dog experiments with expanded polytetrafluroethylene material used as arterial vessels.

1976: ASAIO Journal reports successful chronic HD with an expanded polytetrafluroethylene graft.

1980: Totally implanted ports are used for accessing arterial chemotherapy in long-term treatment. A port 
was designed/developed by one of the authors (F.P., an engineer at Metal Bellows, Inc.). The simple device was quickly adopted in the USA and Europe for long-term parenteral chemotherapy and home total parenteral nutrition.

1980s: Several companies developed hybrid transcutaneous access devices comprising an AV graft and a port (i.e. Hemasite ${ }^{\mathrm{TM}}$ (Renal Systems) and Bently Button (Bently Laboratories)). The port portion protrudes partially out of the skin with the interior portion comprising an AV graft. It provided entry to the bloodstream without cutting tissue which patients welcomed, but complications occurred with rapid and devastating effects (i.e. sudden fatal infections and loss of patency). By the early 1990s, these devices were removed from the market.

1996: Two start-up companies (Vasca and Biolink) were formed to develop totally implanted blood ports for HD treatment. Biolink was founded by the authors. Both companies sold their products for several years (Biolink in Europe and Vasca in the USA) [1]. However, by 2004, both companies left the market for failure to satisfy investor goals.

\section{Assessment and Description of Blood Access - Current Needs}

The present authors continued to seek better access. Vasca's clinical experience combined with Biolink's provided a solid understanding of performance failures as well as successful aspects of implantable HD port systems. For example, both port designs suffered from high bloodstream infection caused by universal biofilm colonization within the blood pathway.

Blood access methods in use today were invented, developed and introduced nearly four decades ago. Although many improvements have occurred, we are still plagued with high morbidity, mortality and aversion by patients. Consumption of large portions of the healthcare budget is required to create and maintain blood access. Patients often reject home dialysis because of fear of access and resist self-accessing.

The following is a critical assessment of current access shortcomings followed by a description of our blood access specifically in terms of current needs.

\section{Arteriovenous Fistula and Arteriovenous Graft}

AVF is the preferred blood access with arteriovenous graft (AVG) the second choice of dialysis providers and nephrology institutions. AVF and AVG comprise a 'short circuit' flow path between a large artery and large vein usually in the arm thereby creating a high flow in the shunt (i.e. 'short circuit'). The shunt becomes the target site to obtain the necessary high withdrawal blood flow to the HD machine enabling short dialysis times to achieve Kt/V requirements. Simultaneously, the cardiac blood flow substantially increases as it now pumps against lower blood circuit resistance. This causes blood flow, pressure levels and flow energy to be redistributed throughout the body. Furthermore, blood not reaching the capillary bed signals the heart to pump even faster. The response is that the heart grows larger to compensate for the anomalous conditions. Many patients exhibit 20\% or greater cardiac flow. These effects have been studied and are discussed below.

After the creation of AVF/AVG, certain morphological events in the body and shunt should occur (i.e. called maturation) before these accesses become usable for HD. This process takes several weeks, but not all accesses reach useful functioning.

Mayo Clinic nephrologists performed a large observational study to assess effects of the Fistula First Initiative [2]. They concluded AVF exhibits failure rates that are among the highest for any elective procedure underscoring the enormity of AVF dysfunction. Their study included 292 AVF constructs resulting in $56 \%$ of them suitable for dialysis and only $41 \%$ still in use at 1 year. High interventions for these constructs over the study period were reported. Their analysis shows better usability and patency associated with shunts comprising larger arteries.

Other reports associate AVF with pulmonary hypertension and high mortality [3]. Several papers describe the cardiovascular effects of AVF associated with structural anomalies of the heart which correlate with high mortality [4-7]. Amerling et al. [8] reviewed patient outcomes with AVF as currently constructed in the US and stated obvious problems with AVF have not been addressed or studied. A recent paper describes underreported fatal AVF hemorrhages [9].

Many patients surveyed describe an adversity to using AVF based on feeling of being stigmatized and a strong sense of vulnerability and loss of confidence with the healthcare team [10].

\section{Catheter Access}

Catheters are considered the least acceptable option for $\mathrm{HD}$ access based on reports of highest mortality, morbidity and costs. Institutional promotion of higher AVF placements has been successful, but has inadvertently in- 


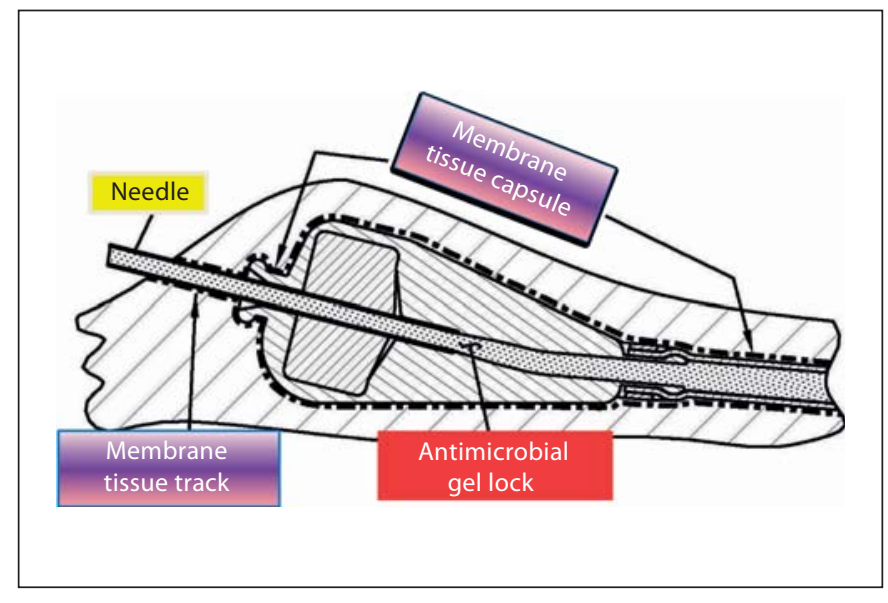

Fig. 1. Access system depicting needle docked with port and lock instilled within the port's luminal passages before needle withdrawal.

creased the proportion of patents with catheters. Current statistics show $82 \%$ of incident patients begin dialysis using catheter access and $18 \%$ of prevalent patients rely on catheters.

The most severe problem associated with catheter use is infection. It is costly to treat and directly associated with death from sepsis. Although antimicrobial lock solutions have demonstrated substantial reductions in catheter-associated infection, the locks are not available in the USA. Another dominate complication of catheters is loss of patency which is correctable. Furthermore, newer catheter designs, tip placement in the right atrium and use of catheter prophylaxis at the time of placement substantially reduce complications of infection and patency. Many patients find catheters diminish selfimage and restrict certain activities (e.g. swimming, bathing).

\section{A New System}

Our system comprises several elements comprising an apparatus, compositions and methods combined into a system. The system is described explicitly in our PCT Patent Application [11] which is currently undergoing examination at the European and US patent offices. The application is publicly available through the patent offices or from the author. The new system/elements are described below and schematically in figure 1 and 2 .

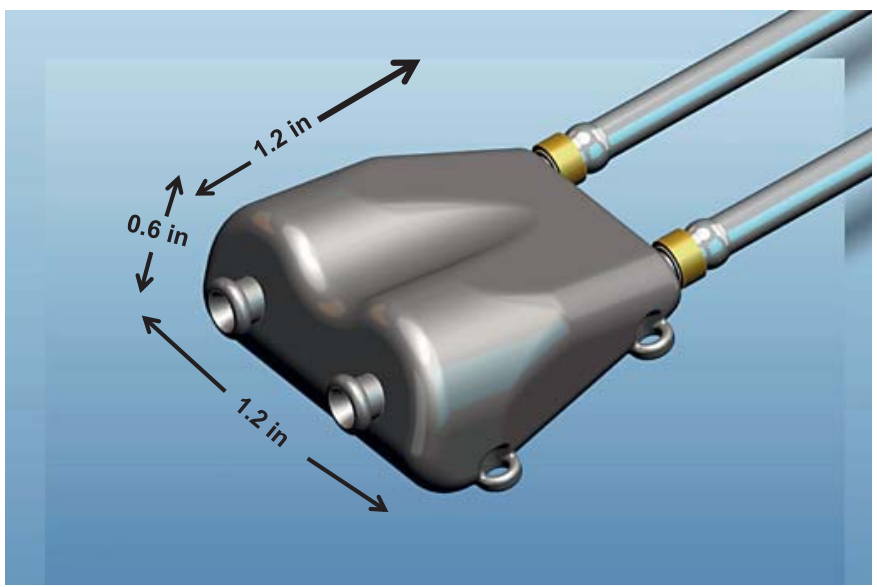

Fig 2. A new port.

\section{Totally Implanted Port}

The port comprises two flow channels comprising novel septum closures, a needle-locking feature and means to fasten the transcutaneous tissue tract (TTT) to the port to maintain alignment. The shape and size (i.e. low profile and small size) reduce tensional stress in tissue overlaying the port. The simple blood closure opens/closes automatically with needle penetration. Hardware is robust utilizing high-reliability design techniques. After port placement in the subcutaneous pocket the surgeon uses a special piercing tool guided by the port's entrance aperture to project a straight line through tissue using a retrograde insertion through the port and precisely establishes a puncture line projected from the port to the outside. The piercing tool is removed and a small tool is inserted along the pierced line to enter the port. This tool is shaped to enable natural encapsulating tissue forming into a tiny rectangle (i.e., approx. $2 \times 0.1$ $\mathrm{mm}$ ) which becomes the TTT.

\section{Transcutaneous Tissue Tract}

TTT is a tissue construct formed naturally around the tool inserted into the port as described above. A surgeon's experience and skill favored with a clear visual field of the port/tissue interface enables the most appropriate port installation to fix the TTT position to accommodate patient accessing. TTT enhances needle guidance and better hygienic conditions than the conventional buttonhole (BH) technique used with AVF. AVF/BH technique relies on clotting of blood within the tract to form a blood seal 
after needle removal requiring several minutes maintaining tissue compression after HD is completed. Current technique for creation of the $\mathrm{BH}$ is often difficult for patients to tolerate as it may incur hit-and-miss puncturing over a few weeks. Several recent reports show higher rates of infection using the $\mathrm{BH}$ technique than the former rotated rope-ladder puncturing method.

TTT has different etiology and morphology than $\mathrm{BH}$. Conventional $\mathrm{BH}$ is formed by the repeated cycles of cutting tissue, bleeding and healing to create a scar tissue morphology which is not optimum in terms of shape or smoothness. Also, $\mathrm{BH}$ tends to not close fully when a needle is extracted. Bleeding into the subcutaneous tissue is intrinsic with AVF accessing but is absent in this port/TTT system. Tissue comprising TTT is the same material which normally forms around implanted devices. It is composed of fibrous connective tissue forming an encapsulating thin membrane which is strong and attached to tissue on the outside. TTT is very smooth and slippery inside and it is shaped to provide a small slit shape duct through subcutaneous tissue. The duct is entered by a blunt non-cutting tube which forces separation of the two sides of the rectangle construction. TTT creation at the time of port placement enables contiguous joining of TTT with the tissue capsule forming around the port. Intrinsic in the access system is application of a lubricious gel prophylaxis which helps guide needle penetration and prevents biofilm colonization. Penetration occurs as the blunt needle tip separates the slit to allow needle insertion without tissue damage or bleeding. TTT closure is by pressure bandage to protect against contamination entering the space within TTT and redundant action of the viscoelastic gel instilled during needle extraction. Primary blood sealing is provided by the port.

\section{Gel Prophylaxis}

Biolink and Vasca trials demonstrated the need for preventing infections associated with ports and catheters. Biolink ports experienced infection complications at the very start of their US pilot trial in 1997. William Costerton (Center for Biofilm Engineering, Montana State University) tested Biolink ports removed from the several patients predicating in the initial Biolink clinical pilot trial (PI Nathan Levin, Beth Israel Hospital, New York City). SEM examination determined that all ports exhibited viable Biofilm on the blood circuit luminal surfaces, regardless of the patient's infection status. Prior research identified biofilm as the cause of most bloodstream infections. Putative understanding is that biofilm fragments shed into the bloodstream causing bloodstream infection to occur if host defense is compromised. Abundant data shows most catheter infections can be prevented by using antibiotic/antimicrobial locks.

Dr. Klaus Sodemann invented a taurolidine lock composition which was used in Biolink's port trial in Europe which greatly reduced port infections. Biolink obtained European commercial approval of the taurolidine lock for catheters and ports in Europe in 2001. This type of liquid lock is available in many countries (e.g. TauroLock ${ }^{\circledR}$; TauroPharm GmbH, Waldbüttelbrunn, Germany). Dr. Sodemann has been using the lock for more than 12 years with extremely low infection rates similar to AVF patients in the USA. European standards for HD treatment recommend use of antimicrobial locks as prophylaxis for HD catheters as well as a means to eradicate biofilm in catheters which have already become infected [13].

Antimicrobial liquid locks indeed show substantial prophylactic effectiveness. This is clearly demonstrated in large numbers of papers over the last 10 years; nearly without exception; showing substantial reduction in catheter-related bloodstream infection. However, Polaschegg discoverered a problem and he showed with theory and experimental data that a considerable amount of the lock spills out in vivo [12]. Loss of lock solution has various consequences including overdosing heparin and consequent bleeding risks, drug interactions, loss of lock protection in the catheter and enablement of blood entering the catheter. Subsequently, Polaschegg developed means to prevent spillage using viscoelastic gels for lock compositions comprising prophylactic locks [14]. The term 'viscoelastic' describes the properties of certain compositions which exhibit solid properties when subjected to low stress and zero strain rate (i.e. elastic) and then transforms to liquid characteristics (i.e. viscosity) when subject to a strain rate after the yield strength is exceeded.

\section{Ancillary Elements}

An integrated bloodline with few connections and an improved streamlined flow path eliminates abrupt flow area changes and dead zones. It incorporates a patientfriendly interface for home treatment including smaller size, better drapeability and added safety. The bloodline assembly comprises the dialyzer and special needle which docks and locks into the port and the tubing lines. This external circuit may be sterilized repeatedly and is more convenient for home patients, especially when integrated into the dialysis machine design. 
A special needle improves upon Biolink's needle assembly by eliminating certain failure modes in the earlier design. Also, the needle is much smaller and incorporates a 'fail-safe' needle dislodgement feature.

\section{Conclusion}

Our system is useful for people who need immediate dialysis as a replacement for catheters, especially for patients not considered likely to develop functional AVF constructs or are susceptible to cardiovascular complications associated with AVF or AVG. Home dialysis patients may especially welcome this system for providing safe and easy accessing and its many patient-friendly attributes. Most importantly, the system helps patients to be self-reliant, improve self-awareness and become rehabilitated. This goal becomes increasingly important as current care for HD is becoming unaffordable in the USA.

The vital features which address patient vital concerns are: (1) no tissue trauma from needle punctures; (2) highly robust with added layers of safety; (3) fail-safe needle dislodgement and no bleeding during treatment; (4) needle connection is easy, accurate, bloodless and painless; (5) correct docking sensed with biofeedback and solid tactile indication from needle stop indicating correct position; (6) needle locks to the port automatically; (7) no bleeding after needle withdrawal at conclusion of HD; (8) global effective prophylaxis; (9) two hands available to make accessing considerably easier; (10) helps avoid need for a partner to make the access connection, and (11) bet- ter quality of life, independence without a partner, (b) less stigmatizing compared to access, (c) no loss of arm and hand function, and (d) there is reduced sense of vulnerability which patients describe with AVF (i.e. (i) it is substantially more robust, (ii) protection from needle dislodgement secures the external blood circuit, and (iii) there is no bleeding after needle removal).

\section{System Development Status}

Proof of principle of major system elements has been performed. Biolink's clinical experience in Europe validates the ability of patients to safely self-access for their dialysis. Effective prophylaxis against catheter-related bloodstream infection has been demonstrated in numerous liquid lock trials. Also, the liquid lock has been used to prevent (or eradicate) subcutaneous needle tract infections with HD ports. Biocompatible viscoelastic gel compositions have been made and tested which demonstrate attributes necessary for catheter locks. These gel compositions may function as a universal carrier/depot for a wide range of antimicrobial agents or other APIs. Biolink's and Vasca's experience demonstrates that patents accept ports from a self-image perspective similar to chemotherapy patients. Lastly, the failures experienced by Biolink and Vasca ports are solved with our system.

\section{Disclosure Statement}

The authors have no conflicts of interest to disclose.

\section{References}

1 Moran JE, Prosl F: Totally implantable subcutaneous devices for hemodialysis access. Contrib Nephrol 2004;142:178-92.

-2 Schinstock CA, Albright RC, Williams AW, et al: Outcomes of arteriovenous fistula creation after the Fistula First Initiative. Clin J Am Soc Nephrol 2011;6:1996-2002.

3 Yu TM, Cheng CH, Shu KH: Influence of access blood flow on pulmonary hypertension in patients undergoing hemodialysis. J Am Soc Nephrol 2008; 19:261A.

4 Iwashima Y, Horio T, Takami Y, et al: Effects of the creation of arteriovenous fistula for hemodialysis on cardiac function and natriuretic peptide levels in CRF. Am J Kidney Dis 2002;40:974-982.
5 Savage MT, Ferro CJ, Sassano A, Tomson CR: The impact of AVF formation on central hemodynamic pressures in chronic renal fail ure patients: a prospective study. Am J Kidney Dis 2002;40:753-759.

6 Ori Y, Korzets A, Katz M, et al: The contribution of an arteriovenous access for hemodialysis to left ventricular hypertrophy. Am J Kidney Dis 2002;40:745-752.

7 Bos WJ, Zietse R, Wesseling KH, Westerhof $\mathrm{N}$ : Effects of arteriovenous fistulas on cardiac oxygen supply and demand. Kidney Int 1999;55:2049-2053.

$>8$ Amerling R, Ronco C, Kuhlman M, Winchester JF: Arteriovenous fistula toxicity. Blood Purif. 2011;31:113-120.

9 Ellingson KD, Palekar RS, Lucero CA, et al: Vascular access hemorrhages contribute to deaths among hemodialysis patients. Kidney Int 2012, E-pub ahead of print.
10 Richard CJ, Engebretson J: Negotiating living with an arteriovenous fistula for hemodialysis. Nephrol Nurs J 2010;37:363-375.

11 Prosl F, Polaschegg HD: Hemodialysis access system. Patent Application. Int Publ No. WO2012/06488 A2, May 2012.

12 Polaschegg HD: Catheter locking solution spillage: theory and experimental verification. Blood Purif 2008;26:255-260.

13 Vanholder R, Canaud B, Fluck R, et al: Catheter-related bloodstream infections: a European view. Nephrol Dial Transplant 2010;25: 1753-1756.

14 Polaschegg HD: Composition for prevention of indwelling device-related infection. European Patent Specification EP1442753 B1, granted February 21, 2007. 